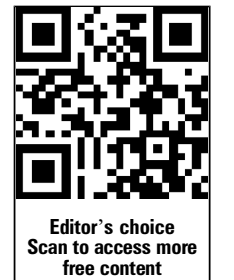

${ }^{1}$ UCT/MRC Research Unit for Exercise Science and Sports Medicine, Department of Human Biology, Faculty of Health Sciences, University of Cape Town, Cape Town, South Africa

${ }^{2}$ Department of Public and Occupational Health, EMGO Institute for Health and Care Research, VU University Medical Center, Amsterdam, The Netherlands ${ }^{3}$ South African Rugby Union (SARU), Cape Town, South Africa

\section{Correspondence to} Dr Wayne Viljoen, South African Rugby Union (SARU), SARU House, 163 Uys Krige Road, Plattekloof, Cape Town, South Africa; waynev@sarugby. co.za

Received 11 July 2013 Revised 20 January 2014 Accepted 24 January 2014 Published Online First 18 February 2014

\title{
An evidence-driven approach to scrum law modifications in amateur rugby played in South Africa
}

\author{
Sharief Hendricks, ${ }^{1}$ Mike I Lambert, ${ }^{1}$ James C Brown, ${ }^{1,2}$ Clint Readhead, ${ }^{3}$ \\ Wayne Viljoen ${ }^{3}$
}

\section{ABSTRACT}

Background In 2012, the South African Rugby Union (SARU) approved a new set of scrum laws for amateur rugby played in the country, to be implemented at the start of the 2013 rugby season. These law changes were primarily based on the relatively high proportion of scrum-related catastrophic injury data collected as part of the BokSmart National Rugby Safety Programme

(BokSmart) over the preceding 4 years (2008-2011).

Aim To describe the scrum-related catastrophic injury data in South Africa over the past 5 years (2008-2012), and to discuss how this evidence justifies the change in the Amateur Scrum Laws to make this aspect of the game safer in South Africa.

Methods Catastrophic injury data were collected through BokSmart at amateur and professional levels, during training and matches over 5 years (2008-2012).

Results The scrum phase accounted for $33 \%$ ( $n=20$ of 60) of all catastrophic injuries between 2008 and 2012. Eighteen of the 20 scrum injuries (90\%) were confirmed as acute spinal cord injuries, with 13 of these being permanent injuries. For the scrum injury mechanisms that were provided $(n=19)$, 'impact on the engagement' was the most frequently reported $(n=11$ of $19,58 \%)$, followed by 'collapsed scrum' ( $n=7$ of 19,37\%) and 'popping out' ( $n=1$ of $19,5 \%)$.

Conclusions Based on these scrum-related catastrophic injury data, a change in the Amateur Scrum Laws of South African Rugby was justified. The main purpose of these scrum law changes is to reduce the number of scrum-related catastrophic injuries in the country, by minimising the opportunity for impact injury and subsequent scrum collapse in amateur rugby in South Africa, thereby making this aspect of the game of rugby safer.

\section{INTRODUCTION}

The scrum (short for scrummage) is a fundamental facet of play in Rugby Union (hereafter 'rugby') and serves to restart the game after a stoppage in play (eg, following a minor infringement such as a forward pass or knock on). The scrum occurs when eight players (collectively referred to as 'forwards') from each opposing team organise themselves in a set formation to physically engage each other and compete for possession of the ball (figure 1). This set formation consists of a front row of three players (a hooker tightly embracing two props on either side-referred to as tight head and loose head props), a second row (2 locks, with heads lodged between the hooker and prop on either side) and a back row of three players (2 flankers on either side of the locks, pushing up against the props, and an eighth man with head between and shoulders pushing up against the locks). During scrum engagement, the forward packs and, more specifically, the front row of each opposing side forcefully engage and interlock with each other, using their heads, necks, shoulders and arms, while maintaining a low body position. The restricted movement and the forceful impact of engagement of the front row players during a scrum has been cause for concern over the years as this places the front row at potential risk of catastrophic cervical spinal injury, should their technique, timing, sequencing or body position be wrong during this forceful engagement. ${ }^{1}{ }^{2}$ Moreover, if the engagement between the opposing scrums is not successful, the scrum may collapse, thereby further exposing front rowers to the added risk of potential catastrophic injury. ${ }^{1}$

Although a catastrophic injury is a rare event in rugby, ${ }^{3}$ for the safety of the players and the continued development and promotion of the sport, all measures need to be considered to prevent these tragic events. ${ }^{4}$ In 2007, law changes to scrums were introduced by the International Rugby Board (IRB) to improve player welfare at scrum time, and to better control and manage the scrum engagement process. ${ }^{6}$ This law applied a four-stage call sequence command by the referee, 'crouch', 'touch', 'pause' and 'engage', to allow for a shorter, more controllable and fixed distance between the two front rows. It was assumed that if the distance between the two front rows was shorter and fixed, the impact forces during engagement would be reduced. Before this law change, players were only commanded to 'engage' when ready from an unfixed distance.

The BokSmart National Rugby Safety Programme (BokSmart) is a national programme implemented on behalf of the South African Rugby Union (SARU) and the Chris Burger/Petro Jackson Player's Fund (CBPJPF). ${ }^{7}$ The goal of the programme is to teach safe and effective techniques, which aim to reduce the incidence and severity of injury. The main focus is to try and lower the number of catastrophic head, neck and spine rugby-related injuries. SARU considers one catastrophic injury as one too many. An intended consequence is to make the game safer for all involved by identifying and coaching specific rugby skills ${ }^{7}{ }^{8}$ associated with both safety and improved player performance.

In 2012, the SARU approved a new set of scrum laws for amateur (club and school) rugby played in the country, to be implemented at the start of the 2013 rugby season. ${ }^{9}$ These law changes were primarily based on the high proportion of scrumrelated catastrophic injury data collected as part of 


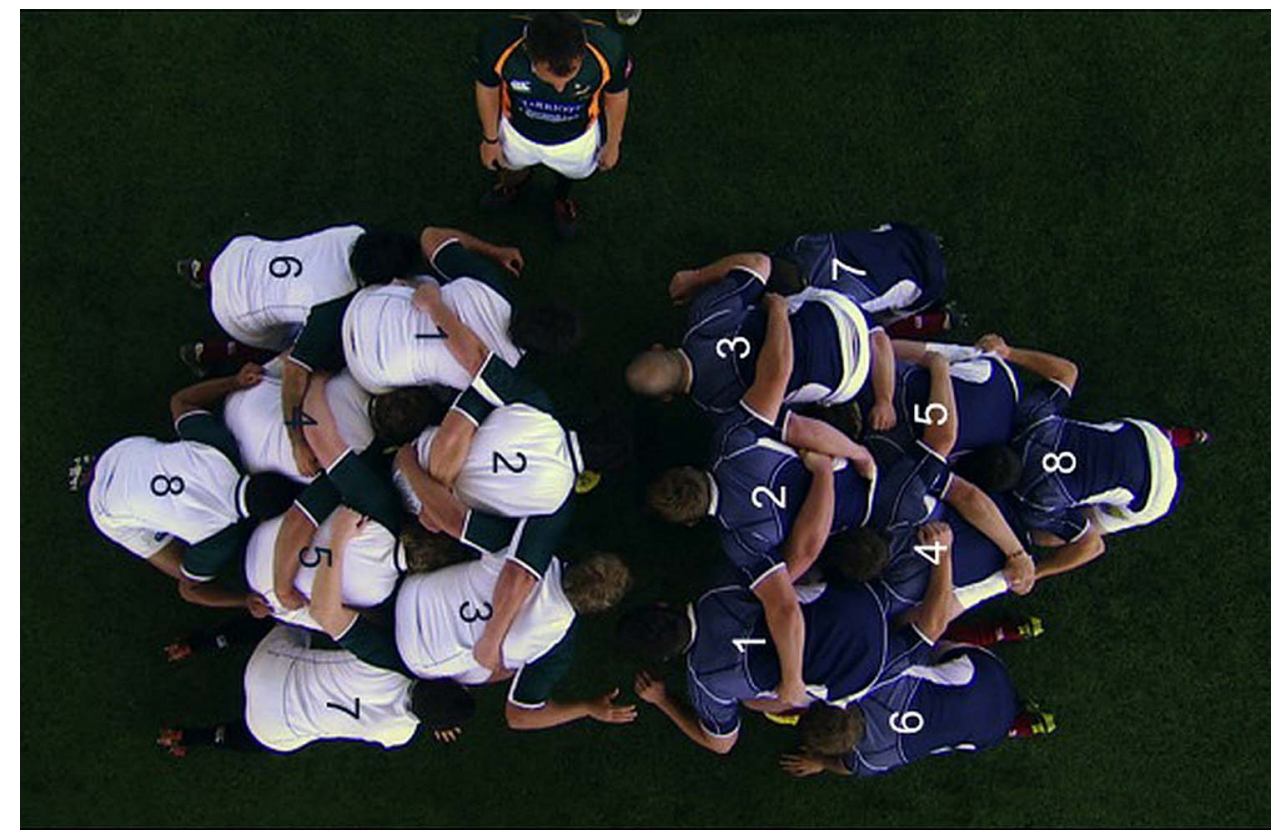

Figure 1 Scrum formation before engagement. Players 1, 2 and 3-front row. Players 4 and 5-second row. Players 6, 7 and 8 -loose forward.

the BokSmart programme over the preceding 4 years (20082011). ${ }^{1}$ The purpose of this paper is to describe the scrumrelated catastrophic injury data in South Africa over the past 5 years (2008-2012), and to discuss how this evidence justifies the change in the Amateur Scrum Laws to make this aspect of the game safer in South Africa.

\section{METHODS}

Data for this study were collected through the BokSmart programme, ${ }^{7}$ which is a joint initiative between the SARU and the CBPJPF. The CBPJPF is a non-profit public benefit organisation, developed to aid players who have been permanently disabled while playing rugby in South Africa. All coaches are aware of the BokSmart Programme and the CBPJPF, and that all catastrophic injuries need to be reported. For all catastrophic injuries in South Africa, BokSmart, via the CBPJPF, employs a full-time Serious Injury Case Manager who collects all the details regarding the injury, and these data are collated into a central database at SARU. Permission to analyse the data was obtained from the SARU and CBPJPF. This paper only describes the scrum-related catastrophic injury data over the past 5 years (2008-2012). That said, it should be noted that data for injuries during the period 2008-2011 have previously been reported on in a general rugby-related catastrophic injury paper. $^{1}$

\section{Catastrophic injury}

BokSmart uses the following definition for recording catastrophic injuries:

Any head, neck, spine or brain injury that is life-threatening or has the potential to be permanently debilitating and results in the emergency admission of a rugby player to a hospital or medical care center.

Injury data were collected at amateur and professional levels, and during training and matches. Catastrophic head, neck and spine injuries were classified as either an acute spinal cord injury (ASCI) or traumatic brain injury (TBI). The definition used for this study included 'near misses' (full recovery expected, ambulant ${ }^{1}$ ).

Information on the following factors related to the scrum injuries were also collected:

- Nature of the injury

- Outcome of injury within 1 month after injury

- Playing position-for example, hooker, prop, lock, loose forward or eighth-man.

- Scrum injury mechanism-for example, scrum engagement, scrum collapse, popping out.

- Subjective comments relating to the cause of the injury/event, either self-reported by the player, coach, referee, family or friends.

- Which team had ball possession at the time of the scrum.

- Coaching received on safe scrum techniques such as body position, setup, scrum engagement and scrum collapse techniques.

- Surface footing at the time of the scrum-good solid footing, medium grip or slippery.

\section{RESULTS}

There were 60 catastrophic head, neck and spine rugby injuries between 2008 and 2012 in South Africa. Eighty-seven per cent $(n=52)$ of these injuries were ASCIs, and the remaining 13\% $(n=8)$ TBIs. Of the 52 ASCIs, 96\% $(n=50)$ occurred at the location of the neck. The tackle $(n=28$ of $60,47 \%)$ and scrum $(n=20$ of $60,33 \%)$ phases accounted for the greatest number of injuries overall. The scrum phase had a greater proportion of permanent injury outcomes $(n=14$ of $20,70 \%)$ than the tackle $(n=16$ of $28,57 \%)$. Eighteen of the 20 scrum injuries $(90 \%)$ were confirmed as ASCI (table 1). For the remaining two cases, no official classification was confirmed. Ninety-five per cent $(n=19)$ of the scrum injuries were sustained at the amateur level: $60 \%(n=12)$ at club level, $35 \%(n=7)$ at school level. One injury (5\%) was sustained at a professional level. Eighty-five per cent of injuries $(n=17)$ occurred during matches 
Table 1 Nature of scrum acute spinal cordinjuries and outcome of injury within 1 month after the injury (2008-2012)

\begin{tabular}{|c|c|c|}
\hline Nature of injury & $\mathrm{N}$ & Outcome of injury within 1 month after injury \\
\hline C2/C3 dislocation & 1 & Residual damage but can walk without assistive devices \\
\hline C2/C3 incomplete & 1 & No apparent residual damage and full recovery expected \\
\hline C3/C4 fracture dislocation & 1 & Quadriplegia and wheelchair users \\
\hline C4/C5 dislocation & 1 & Residual damage but can walk without assistive devices \\
\hline C4/C5 fracture dislocation & 3 & $\begin{array}{l}\text { No apparent residual damage and full recovery expected } \\
\text { Residual damage but can walk without assistive devices } \\
\text { Quadriplegia and wheelchair users }\end{array}$ \\
\hline $\mathrm{C} 4 / \mathrm{C5}$ fracture dislocation and $\mathrm{C} 4$ central cord syndrome & 1 & Residual damage but can walk without assistive devices \\
\hline C4/C5 bifacet fracture dislocation & 1 & Quadriplegia and wheelchair users \\
\hline C5 lamina and spinal process fracture & 1 & Residual damage but can walk without assistive devices \\
\hline C5/C6 fracture dislocation & 2 & Quadriplegia and wheelchair users \\
\hline C5/C6 bifacet dislocation & 2 & $\begin{array}{l}\text { Quadriplegia and wheelchair users } \\
\text { Not provided }\end{array}$ \\
\hline C5/C6 fracture dislocation with considerable spinal cord damage & 1 & Quadriplegia and wheelchair users \\
\hline C6 fracture & 1 & Not provided \\
\hline C6/C7 bilateral facet dislocation & 1 & Residual damage but can walk without assistive devices \\
\hline C6/C7 dislocation & 1 & Quadriplegia and wheelchair users \\
\hline L3/L4 disc prolapse & 1 & Not provided \\
\hline $\mathrm{T} 2 / \mathrm{T} 3$ plate fractures & 1 & No apparent residual damage and full recovery expected \\
\hline
\end{tabular}

and $10 \%(n=2)$ during training. For only one of the cases was the scrum detail at the time of injury not provided.

Ninety-five per cent (19 of 20 ) of all scrum-related injuries were sustained by the front row (hooker $\mathrm{n}=13,65 \%$, props $\mathrm{n}=6,30 \%)$. All 13 scrum-related injuries to the hooker occurred at the site of the neck. Five of the six scrum-related injuries to the prop occurred at the neck (one occurred at the lumbar spine). In one isolated case, a lock sustained a neck injury in the scrum; this is the only recorded catastrophic scrum injury until now involving a position other than the front row that the authors are aware of. Injury mechanisms were provided for all but one of the cases. For the scrum injury mechanisms that were provided, 'impact on the engagement' was the one reported most frequently ( $\mathrm{n}=11$ of $19,58 \%)$, followed by 'collapsed scrum' ( $\mathrm{n}=7$ of $19,37 \%)$ and 'popping out' ( $\mathrm{n}=1$ of 19 , $5 \%)$.

The following comments, relating to the cause of the injury, were either self-reported by the player or the coach, referee, family or friends, who were present on the field at the time of the injury. It is important to note that these comments were completely subjective, reported verbatim, and were at times peripheral to the mechanism of injury $(n=18,2$ missing comments). Comments are categorised into 'impact on the engagement', 'collapsed scrum' and 'popping out'.

\section{Impact on engagement}

- The scrum was too far apart; the loosehead did not engage properly.

- At the impact of the scrum.

- Space between tighthead and hooker opened up, pressure of locks came through; he was not ready to engage and space closed up before he could adjust, followed by impact.

- Was busy with the scrum binding and the opposition hooker went in too early and hit him on the front of his neck, below the chin. His body went lame and he found himself hanging between the props. Early engagement by the opposition.
- At the first scrum, the referee called and binding happened. I felt my head hit the opposition hooker and prop. I felt my body lose power. When the scrum was cleared, he remained on the floor, unable to move for about $10 \mathrm{~s}$. Experienced burning in body and pain in his neck. He knew that something was seriously wrong.

- On engagement of the scrum, I started feeling weak and then collapsed. After a reset scrum, the injury occurred on engagement.

- His opponent in the scrum engaged too early and his head crashed against the opponent's shoulders. Opponent engaged before the ref indicated.

- During the scrum, the referee was calling very fast. The other hooker's head was in line against mine on crouch; on touch engage, he tried to loosen his head, opposition hooker, but it was too late as he engaged, and the props pulled him down. All players went in except player went up.

- On engagement, the tighthead prop stood up while the rest of the players engaged. The hooker did not get up.

- Got late in the scrum position (Got into the scrum position too late).

- At the time I was busy talking to the referee telling him that my locks are not in, the team engaged.

- Before engagement, the prop pulled the player to him, and his head hit against the shoulder of the opposition. He collapsed and couldn't talk properly. Incorrect binding of head and shoulders.

\section{Collapsed scrum}

- The referee stopped the scrum engagement, but the opposition pack continued with the hit. Injury occurred when the scrum collapsed and the players fell on top of him.

- The scrum collapsed backwards. First scrum of the game.

- The third scrum collapsed, fell onto the player. Felt that the referee should not have called for a third scrum.

- The scrum collapsed and the players continued pushing over the fallen players. After the scrum collapsed, some players 
still went over the fallen players, and the player was thus stepped on as well.

- The scrum collapsed, one prop fell on one side and the scrum turned; then his neck was injured.

\section{Popping out}

- The scrum lifted but could not get his head out.

From 2008 to 2011, the scrum phase accounted for a gradually increasing number and high proportion of match-related ASCIs, which largely resulted in permanent disability $(n=13$; figure 2$)$. In 2012, only one ASCI was recorded. In all the years, the overwhelming majority of match scrum-related injury outcomes have been permanent (with remaining neurological deficit, quadriplegia or death), with the hooker being increasingly overrepresented. The hooker position, being only one position in the team, suffered 9 of the 13 permanent injury outcomes in the scrum (70\%). The neck was injured during all 9 permanent injuries to the hooker.

Ten of the 20 scrum-related catastrophic injury cases provided information on who put the ball into the scrum at the time of injury. Of these 10 cases, $60 \%$ of injuries occurred on 'opposition put-in into the scrum', and $40 \%$ of injuries occurred on 'own put-in'. Nineteen of the 20 catastrophic injury cases also provided information on having or not having received coaching on safe scrumming. Ninety-five per cent reported having received coaching on safe scrum techniques, and safe scrum engagement techniques. Sixty-eight per cent reported having received coaching on safe scrum collapse techniques. Seventeen (out of 20) of the scrum-related injuries also provided information on the field conditions at the time of the scrum. Forty-seven per cent reported having a good solid footing at the time of the scrum, 35\% reported having a medium grip and $18 \%$ reported having slippery conditions.

\section{DISCUSSION}

BokSmart aims to educate coaches, administrators and players on safe and effective rugby techniques in an attempt to reduce the incidence and severity of catastrophic head, neck and

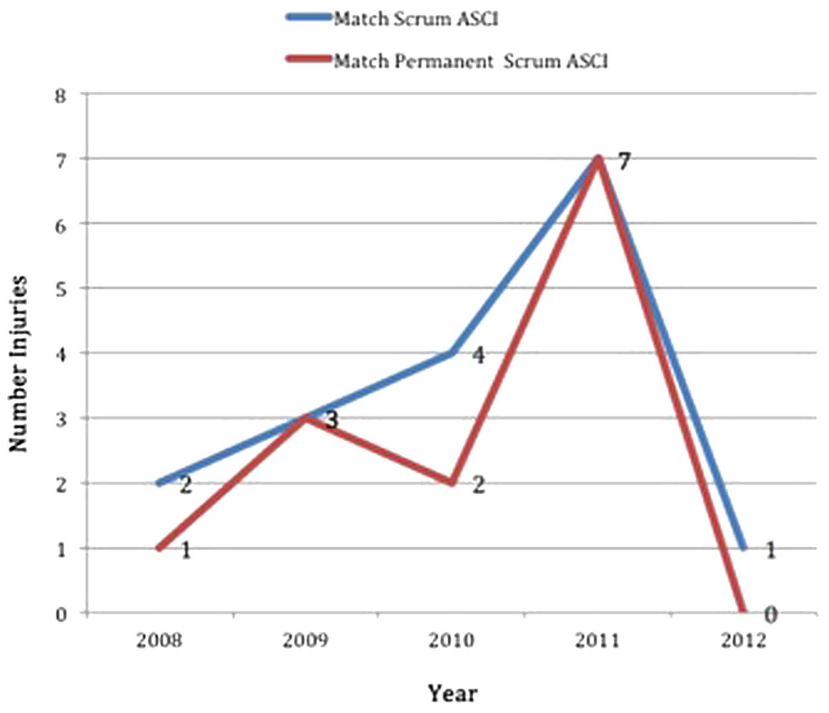

Figure 2 Pattern of match-related scrum acute spinal cord injuries (ASCl) between 2008 and 2012 in South Africa, including permanent injury trends. spine-related rugby injuries. ${ }^{78}$ This education is also important for improved player performance, and for the continued growth, development and promotion of rugby in South Africa. Given the initial systematic increase and relatively high proportion of scrum ASCI in South Africa, ${ }^{1}$ and the increased level of severity (permanent outcomes) of scrum-related ASCI as opposed to other facets of the game such as the tackle situation, ${ }^{10}{ }^{11}$ it became apparent that BokSmart's current interventions, albeit supported by research evidence, ${ }^{12}{ }^{13}$ would not necessarily be able to have an immediate impact in reducing the number of these catastrophic scrum-related injuries. The reason for this was that it would take a reasonable amount of time to filter the necessary and appropriate education and knowledge to all stakeholders of the game in South Africa, that is, to the approximately 47000 coaches and referees, and then for them to implement and transfer this knowledge to the players that they have control over. The uptake, adoption and implementation of these processes and protocols down to grass-roots levels takes time and based on the pattern of the scrum-related injury data collected in South Africa, there would, theoretically, be an unacceptable amount of these injuries that potentially could have been prevented. Using a similar education model to that developed by RugbySmart New Zealand, ${ }^{12}{ }^{13}$ BokSmart realistically only expected to have a noticeable impact on catastrophic injuries following a 5-10-year period of implementation, once the necessary safety protocols, policies and interventions had received sufficient attention within the national rugby landscape and amateur structures. Considering this, and additional evidence available from studies in France, ${ }^{11}$ Britain and Ireland ${ }^{10}$ on cervical spine injuries during the scrum, BokSmart had to implement alternative measures which could potentially have an immediate impact on making the scrum safer. ${ }^{14}$ Amateur rugby was targeted as most of the players in South Africa are amateur, and the increases in, and numbers of, scrum-related injuries were observed at this level.

Overall, the tackle (47\%) accounted for more ASCI than the scrum (33\%). This finding is comparable to similar studies documenting serious spinal injuries. ${ }^{10} 12$ That said, the scrum had a greater proportion of permanent injury outcomes than the tackle. Collectively, these findings echo reports by MacLean and Hutchison ${ }^{10}$ that noted that even though the scrum has proportionally less frequent serious neck injuries than the tackle, the neck injuries sustained in the scrum are more severe and are associated with an increased risk of permanent spinal cord injury.

The scrum-related ASCIs were predominantly associated with the scrum engagement and scrum collapse. Many of these scrum collapse injuries could potentially also be directly linked to the scrum engagement process. Unlike the tackle, the scrum is essentially more amenable to a structured intervention, ${ }^{11} 12$ as the scrum is a structured phase of play, and the scrum engagement can be technically controlled and managed partially by the referee. Therefore, a working group of experts consisting of representatives from medical, coaching and refereeing disciplines was established to explore intervention options. From this working group, a long-term player development pathway was then proposed to SARU regarding the scrums. This model recommended appropriate progressions in the level of impact on engagement, prebinding of the front rows to improve stability and advancements of the scrum contest after the engagement. The biomechanical demands of the scrum at different playing levels provide further support for such a progressive model. ${ }^{15}$ In particular, law modifications to the scrum engagement sequence were addressed. These modifications considered 
the two main issues of the scrum engagement and collapse without compromising scrum performance, and keeping within the essence of what defines rugby union. These recommendations introduced techniques of prescrum binding before engagement and progressing from passive engagement at the younger age-groups, to active engagement with a reduced impact and from a closer distance between the front rows, to the current sequence of engagement being practised worldwide. Also, a new scrum call sequence of 'crouch', 'bind' and 'scrum' was recommended at the lower levels as the calls best described the technical associations and requirements of each phase of the scrum contest, which would potentially also improve learning and skill transfer. More specifically, at the under 6 to under 7 level, a three-man uncontested scrum is used. At the under 8 level, a five-man uncontested scrum is introduced. When full 15 's are added at the under 9 level, a full eight-man scrum is used, but remains uncontested. Full eight-man, contested scrumming (pushing allowed in the scrum to a maximum of $1.5 \mathrm{~m}$ ) with passive engagement and prescrum binding follows from the under 10 to the under 16 level. From the under 18 and under 19 schoolboy levels, active engagement with prescrum binding is introduced, and is applied up until the second highest Amateur Senior Club rugby division. The distance between opposing front rows, pre-engagement, is significantly reduced, to where the ears of the opposing front rows are in a straight line across the mouth of the scrum or 'ear-to-ear'. This is an abridged progression from passive engagement to the full engagement currently performed at the senior professional level. This adjustment to engagement of the scrum at the amateur level has the potential to lower the risk of catastrophic injury upon engagement and subsequent scrum collapse. Active engagement, as per the IRB format of engagement at the time, is allowed at the highest Amateur Senior Club rugby division only and all adult Provincial, Super Rugby and National levels. In short, these main scrum law modifications were proposed and accepted by SARU for junior and senior amateur levels in South Africa. These modified amateur laws are aimed at lowering the impact of engagement, reducing the number of scrum collapses, and progressively introducing players to proper and safe scrum technique. Although every player will not develop into a national or provincial level front ranker, every front row player should be well coached, well managed, technically accurate and safe at scrum time, as advocated by the training guidelines of BokSmart.

The above mentioned laws have been in practice since the beginning of the South African 2013 rugby season (start of January). Since these laws were introduced in South Africa, the IRB recommended the implementation of similar law changes to be tried globally for 1 year during the $2013 / 2014$ season. ${ }^{16}$ Accordingly, these laws have been in place at all levels in the Northern Hemisphere since September 2013, the start of the Northern Hemisphere season. Also, recent work by the University of Bath Rugby Science research group in partnership with the IRB on the biomechanics of scrumming will advance our current understanding of the physical and technical requirements of different engagement techniques. ${ }^{15} 17$

In conclusion, this paper demonstrates how prospectively collected and scrum-related catastrophic injury data provide evidence to justify a change in the Amateur Scrum Laws of South African Rugby. The main purpose of these scrum law changes are aimed at reducing the number of scrum-related catastrophic injuries in the country, thereby making this aspect of the game of rugby safer.

\section{What this study adds?}

- Detailed description of scrum-related catastrophic injuries over 5 years.

- How scrum-related catastrophic injury data were used to change the amateur laws of rugby played in South Africa.

\section{How might it impact on clinical practice in the near}

\section{future?}

- How the collection of injury and injury mechanism data in practice can be used to make rugby safer.

- How law changes to certain areas of rugby can reduce the risk of injury.

- A reduction in catastrophic injury in rugby players.

Acknowledgements The authors would like to thank the board of the Chris Burger/Petro Jackson Players' Fund and especially the former Springbok captain, Morne Du Plessis, for his foresight in initiating the fund. The authors would also like to thank Ms Gail Ross, in her current position as Serious Injury Case Manager, for her dedication and effort in collecting the information that has been presented in this study.

Contributors SH, MIL, JCB, CR and WV were involved in conceptualising the manuscript. Data were analysed by SH, MIL, WV and JCB. All authors edited the manuscript extensively, which was drafted by SH originally.

Competing interests None.

Ethics approval The study was approved by the Human Research Ethics Committee of the University of Cape Town.

Provenance and peer review Not commissioned; externally peer reviewed.

Data sharing statement The raw data of absolute injury numbers per year are freely available at http://www.boksmart.com. For obvious reasons, these raw data are depersonalised.

\section{REFERENCES}

1 Brown JC, Lambert MI, Verhagen $\mathrm{E}$, et al. The incidence of rugby-related catastrophic injuries (including cardiac events) in South Africa from 2008 to 2011: a cohort study. BMJ Open 2013;3:e002475.

2 McIntosh AS. Preventing head and neck injury. Br J Sports Med 2005;39:314-18.

3 Fuller CW. Catastrophic injury in Rugby Union. Sports Med 2008;38:975-86.

4 Quarrie KL, Cantu RC, Chalmers DJ. Rugby union injuries to the cervical spine and spinal cord. Sports Med 2002;32:633-53.

5 Donaldson A, Poulos RG. Planning the diffusion of a neck-injury prevention programme among community rugby union coaches. Br J Sports Med 2014; $48: 151-9$

6 Gianotti S, Hume PA, Hopkins WG, et al. Interim evaluation of the effect of a new scrum law on neck and back injuries in rugby union. Br J Sports Med 2008;42:427-30.

7 Viljoen W, Patricios J. BokSmart-implementing a National Rugby Safety Programme. Br J Sports Med 2012;46:692-3.

8 Posthumus M, Viljoen W. BokSmart: safe and effective techniques in rugby union. S Afr J Sports Med 2008;20:64.

9 South African Rugby Union. BokSmart. boksmart.sarugby.co.za. http://boksmart. sarugby.co.za/news/article/130214/New_scrum_laws_for_schools_and_clubs_in_SA (accessed 12 Jun 2013).

10 MacLean JG, Hutchison JD. Serious neck injuries in U19 rugby union players: an audit of admissions to spinal injury units in Great Britain and Ireland. $\mathrm{Br} J$ Sports Med 2012;46:591-4.

11 Bohu Y, Julia M, Bagate $C$, et al. Declining incidence of catastrophic cervical spine injuries in French rugby: 1996-2006. Am J Sports Med 2009;37: 319-23.

12 Quarrie KL, Gianotti SM, Hopkins WG, et al. Effect of nationwide injury prevention programme on serious spinal injuries in New Zealand rugby union: ecological study. BMJ 2007;334:1150. 
13 Gianotti SM, Quarrie KL, Hume PA. Evaluation of RugbySmart: a rugby union community injury prevention programme. J Sci Med Sport 2009;12:371-5.

14 Gielen AC. Application of behavior-change theories and methods to injury prevention. Epidemiol Rev 2003;25:65-76.

15 Preatoni $\mathrm{E}$, Stokes $\mathrm{KA}$, England ME, et al. The influence of playing level on the biomechanical demands experienced by rugby union forwards during machine scrummaging. Scand J Med Sci Sports 2013;23:e178-84.
16 International Rugby Board. News and Media. New scrum process approved for global trial. http://www.irb.com/newsmedia/mediazone/pressrelease/ newsid $=2066642$.html (accessed 26 Jun 2013)

17 University of Bath. Department of Health, Rugby Science Research Group Annual Report 2012-2013. http://www.bath.ac.uk. http://www.bath.ac.uk/health/ documents/rugby-science-annual-report-2012-13.pdf (accessed 13 Sep 2013).

$\underline{\underline{w}}$ 